\title{
Prevalência de Síndrome Metabólica e associação com estado nutricional em adolescentes
}

\author{
Prevalence of Metabolic Syndrome and associations with \\ nutritional status in adolescents
}

\author{
Myrla Gabriela Mendes ${ }^{1}$ (D), Larisse Monteles Nascimento² (D), \\ Keila Rejane Oliveira Gomes ${ }^{3}$ (D), Regilda Saraiva dos Reis Moreira-Araújo² (D), \\ Malvina Thais Pacheco Rodrigues ${ }^{2}$ (D), Telma Maria Evangelista de Araújo ${ }^{4}$ (D), \\ Karoline de Macedo Gonçalves Frota² (D)
}

\begin{abstract}
Resumo
Introdução: A síndrome metabólica é uma desordem multifatorial relacionada, principalmente, com a deposição de gordura abdominal e as alterações da homeostase da glicose e/ou resistência à insulina. Estudos demonstram associações entre o excesso de peso na infância e as taxas de morbimortalidade elevadas na vida adulta; portanto, a presença de alterações metabólicas na infância e/ou na adolescência pode contribuir para um perfil de saúde desfavorável ao futuro dos indivíduos. Objetivo: Determinar a prevalência de síndrome metabólica e verificar sua associação com estado nutricional em adolescentes. Método: Estudo transversal com adolescentes entre 14 e 19 anos de escolas públicas e particulares. Realizou-se avaliação antropométrica, incluindo o índice de massa corporal e o perímetro da cintura, e foram determinados os parâmetros bioquímicos e a pressão arterial. Para o diagnóstico da síndrome metabólica, utilizou-se de critérios da National Cholesterol Education Program's Adult Treatment Panel III (NCEP/ATP III). As características antropométricas e bioquímicas foram expressas como média e desvios-padrão e comparadas entre os sexos pelo teste t de Student, enquanto, para análise de risco, utilizou-se da regressão logística binária. Resultados: Dos 327 adolescentes investigados, 59,9\% eram meninas e 65,7\% estudavam em escola pública. A prevalência de síndrome metabólica foi de $7 \%$, enquanto a presença de pelo menos um critério diagnóstico da síndrome esteve presente em $90,8 \%$ da amostra. Ainda, observou-se associação significativa entre a obesidade e a síndrome metabólica, apontando um maior risco. Conclusão: Os adolescentes tiveram uma importante prevalência de síndrome metabólica, apresentando precocemente componentes dessa patologia. Além disso, foi observada associação com a obesidade, evidenciando um maior risco para aqueles que possuem essa condição; portanto, intervenções nutricionais direcionadas à população jovem devem ser promovidas no intuito de melhorar os hábitos alimentares e o estilo de vida.
\end{abstract}

Palavras-chave: prevalência; síndrome metabólica; obesidade; adolescentes.

\footnotetext{
Abstract

Background: Metabolic syndrome is a multifactorial disorder mainly related to abdominal fat deposition and changes in glucose homeostasis and / or insulin resistance. Studies show associations between childhood overweight and high morbidity and mortality rates in adulthood, so the presence of metabolic changes in childhood and / or adolescence may contribute to an unfavorable

${ }^{1}$ Departamento de Nutrição, Centro de Ciências da Saúde, Universidade Federal do Piauí, Campus Ministro Petrônio Portela - Teresina (PI), Brasil.

${ }^{2}$ Programa de Pós-graduação em Saúde e Comunidade, Centro de Ciências da Saúde, Universidade Federal do Piaúí, Campus Ministro Petrônio Portela Teresina (PI), Brasil.

${ }^{3}$ Colégio Técnico de Teresina, Universidade Federal do Piauí, Campus Ministro Petrônio Portela - Teresina (PI), Brasil.

${ }^{4}$ Departamento de Enfermagem, Centro de Ciências da Saúde, Universidade Federal do Piauí, Campus Ministro Petrônio Portela - Teresina (PI), Brasil. Trabalho realizado em escolas da rede pública e particular do ensino médio - Teresina (PI), Brasil.

Endereço para correspondência: Karoline de Macedo Gonçalves Frota - Programa de Pós-Graduação em Alimentos e Nutrição, Centro de Ciências da Saúde Universidade Federal do Piauí, Av. Universitária - Ininga - CEP: 64049-550 - Teresina (PI), Brasil - Email: karolfrota@ufpi.edu.br

Fonte de financiamento: Coordenação de Aperfeiçoamento de Pessoal de Nível Superior (CAPES).

Conflito de interesses: nada a declarar.
} 
health profile to the future health of individuals. Objective: To determine the prevalence of metabolic syndrome and to check its association with nutritional status in adolescents. Method: Cross-sectional study with adolescents from public and private schools with ages ranging from 14 to 19 years was carried out. It was performed an anthropometric assessment, including the determination of Body Mass Index and Waist Circumference, besides that the determination of biochemical parameters and blood pressure was also collected. In order to diagnose Metabolic Syndrome, it was used the National Cholesterol Education Program's Adult Treatment Panel III (NCEP/ATP III) criteria. The anthropometric and biochemical characteristics were expressed as average and standard deviations, and compared between the genders by the Student's t-test, while for the analysis of risk was used binary logistic regression. Results: Of total 327 interviewed adolescents, $59.9 \%$ of them were female, $65.7 \%$ were studying in public schools. The prevalence of metabolic syndrome was $7 \%$, while the presence of at least one diagnostic criterion of this syndrome was present in $90.8 \%$ of the sample. Moreover, obesity was significantly associated with metabolic syndrome, indicating a greater risk. Conclusion: Adolescents have a high prevalence of metabolic syndrome, showing early components of this pathology, which is still associated with obesity, highlighting a greater risk for those who present this condition. Therefore, nutritional interventions directed at the young population should be promoted, with the purpose of improving eating habits and lifestyle.

Keywords: prevalence; metabolic syndrome; obesity; adolescents.

\section{INTRODUÇÃO}

A síndrome metabólica (SM) é um conjunto de características metabólicas inter-relacionadas que estão ligadas ao desenvolvimento de doenças cardiovasculares (DCV) e diabetes ${ }^{1}$. A obesidade constitui um dos mais importantes problemas de saúde pública, apresentando risco elevado de surgimento precoce das complicações associadas ao excesso de gordura corporal ${ }^{2}$. O acúmulo de gordura geralmente está relacionado à presença de hipertensão e alterações metabólicas, como aumento dos níveis de triglicerídeos e de glicose e baixos níveis de HDL-c (lipoproteínas de alta densidade) ${ }^{3}$. À medida que a prevalência de obesidade continua a aumentar na população, aumenta-se também a prevalência da SM em adolescentes ${ }^{4}$.

A prevalência de SM na população mundial é de $25 \%$, sendo responsável por $7 \%$ da mortalidade global e por $17 \%$ dos óbitos ligados às DCV ${ }^{5}$. Em relação a dados publicados na literatura, são reportadas taxas de prevalência de $\mathrm{SM}$ em adolescentes variando entre $2,5 \%$ e $22,2 \%$, dependendo da população e do critério adotado para diagnóstico da síndrome. Evidências demostram nesse grupo associação significativa entre condicionantes da SM e fatores de risco comportamentais, sedentarismo e obesidade abdominal, ressaltando a importância do diagnóstico precoce ${ }^{8}$.

A NCEP/ATP III ${ }^{9}$ definiu a SM em três ou mais critérios como: obesidade abdominal apresentando perímetro da cintura $\geq$ percentil 90 para idade e sexo; triglicerídeos elevados superior a $150 \mathrm{mg} / \mathrm{dL}$; baixos níveis de HDL-C, apresentando em homens HDL-c menor que $40 \mathrm{mg} / \mathrm{dL}$ e nas mulheres menor que $50 \mathrm{mg} / \mathrm{dL}$; elevada pressão arterial (PA), com valor igual ou superior a 135/85 $\mathrm{mmHg}$, ou uso de algum medicamento para reduzir a pressão arterial; e elevada glicemia de jejum, com valor igual ou superior a $110 \mathrm{mg} / \mathrm{dL}$. Alguns estudos realizados com adolescentes utilizaram critérios análogos da NCEP/ATP III ${ }^{9}$, como os de Cantalice et al. ${ }^{10}$ e Rosini et al. ${ }^{11}$, visto que não há um consenso sobre a definição dos pontos de corte para determinação dos componentes da SM nesse grupo ${ }^{12}$.

Nesse sentido, ressalta-se a importância do surgimento precoce dos componentes da SM, pois, quando presentes na adolescência, podem contribuir para o desenvolvimento dessa patologia. Portanto, o objetivo desta pesquisa foi determinar a prevalência de SM e sua associação com obesidade em adolescentes.

\section{MÉTODO}

Estudo de delineamento transversal realizado com escolares, na faixa etária de 14 a 19 anos, matriculados na rede pública e particular do ensino médio no município de Teresina, Piauí, no ano de 2016. Os dados analisados nesta pesquisa procedem de um projeto-base intitulado "Saúde na escola: diagnóstico situacional do ensino médio", da Universidade Federal do Piauí (UFPI). O projeto foi aprovado pelo Comitê de Ética em Pesquisa da UFPI ( ${ }^{\circ}$ 1.495.975) e pela Secretaria de Educação e Cultura do Piauí (SEDUC), de acordo com as Resoluções n 196/96 e no 466/2012 do Conselho Nacional de Saúde (CNS) ${ }^{13}$. Os estudantes e os pais ou responsáveis assinaram o Termo de Assentimento Livre Esclarecido e o Termo de Consentimento Livre Esclarecido, respectivamente, quando foi o caso.

As escolas foram selecionadas por amostragem probabilística ${ }^{14}$ com estratos por tipo de gestão, área geográfica e porte, em que foram sorteadas uma escola pública e uma particular segundo o porte - pequeno (até 115 alunos), médio (de 116 a 215 alunos) e grande (mais de 216 alunos) -, definido pelo pesquisador e considerado apenas para esta pesquisa, e para área geográfica correspondente a cada Gerência Regional de Ensino (GRE), totalizando 12 escolas públicas e 12 particulares.

Os adolescentes foram selecionados por amostragem probabilística estratificada proporcional ${ }^{14}$. Utilizou-se do programa Epi Info 6.04d (Centers for Disease Control and Prevention, Atlanta, Estados Unidos) para o cálculo da amostra mínima, partindo do universo de 40.136 de estudantes do ensino médio de escolas públicas estaduais e particulares, de acordo com o Censo Escolar de 2014 $4^{15}$, adotando-se intervalo de confiança de $95 \%$, prevalência de $17,1 \%$ de sobrepeso ${ }^{16}$, precisão de $5 \%$, efeito de desenho de 1,4 e significância de $5 \%{ }^{17}$. A amostra mínima considerada para esse recorte do estudo 
base foi de 304 adolescentes. Em razão das possíveis perdas de casos durante a coleta de dados, sortearam-se $10 \%$ a mais da amostra de cada escola, constituindo uma amostra final de 334 adolescentes.

A amostra nas escolas sorteadas foi distribuída proporcionalmente ao número de adolescentes por tipo de gestão das escolas e porte, e, em cada escola sorteada, distribuiu-se a amostra a ela destinada por sorteio proporcional à série, ao sexo e à idade.

Para análise das variáveis antropométricas, os adolescentes foram pesados com auxílio de balança portátil de escala eletrônica (SECA ${ }^{\circledR}$, modelo 803, Hamburgo, Alemanha) com precisão de 100 g. A altura foi medida utilizando um estadiômetro $\left(\right.$ SECA $^{\circledR}$, modelo messband 206, Hamburgo, Alemanha), com precisão de $0,1 \mathrm{~cm}$. O índice de massa corporal (IMC) foi expresso em escore $Z$ e classificado utilizando pontos de corte estabelecidos pela Organização Mundial de Saúde ${ }^{18}$.

A medida da circunferência da cintura (CC) foi obtida segundo a técnica de Callaway et al. ${ }^{19}$, utilizando-se de fita métrica inelástica $\left(\mathrm{SECA}^{\circledR}\right.$, modelo 201, Hamburgo, Alemanha) com precisão de $0,1 \mathrm{~cm}$, no qual a classificação foi realizada de acordo a curva de Fredriks et al. ${ }^{20}$. Foram realizadas três medidas consecutivas e uma média delas para a maior confiabilidade dos dados.

A medida da pressão arterial (PA) foi realizada conforme a metodologia estabelecida pela $7^{\text {a }}$ Diretriz Brasileira de Hipertensão Arterial ${ }^{21}$, com auxílio de um esfigmomanômetro aneroide (Durashock Welch Allyn-Tycos ${ }^{\circledR}$, Nova Iorque, Estados Unidos, Modelo DS-44). Consideraram-se as médias de duas aferições de pressão arterial sistólica (PAS) e pressão arterial diastólica (PAD) em um primeiro momento e após 5 minutos de descanso ${ }^{22}$. Se a PA diferisse mais que $5 \mathrm{mmHg}$ entre as medidas, duas medidas adicionais eram realizadas, e a média delas era utilizada para a análise ${ }^{23}$. Classificaram-se como normotensos os adolescentes com a PAS e a PAD menor que o percentil 90 para sua estatura, relativo ao sexo e à idade ${ }^{19}$.

Amostras de $5 \mathrm{~mL}$ de sangue foram colhidas, após jejum de 12 horas, por enfermeiros nas dependências das escolas, sendo realizada a determinação dos lipídios séricos e da glicemia segundo o método enzimático colorimétrico, utilizando-se kits da Labtest ${ }^{\circledR}$. Para calcular a LDL-c, utilizou-se da fórmula de Friedwald ${ }^{24}$. Os valores de lipídios séricos foram classificados segundo a V Diretriz Brasileira de Dislipidemias e Prevenção da Aterosclerose ${ }^{25}$, e a glicose, de acordo com os critérios definidos pela American Diabetes Association ${ }^{26}$, os quais são utilizados para o diagnóstico da SM juntamente com a definição proposta pela NCEP/ATP III $^{9}$ para adolescentes com idade entre 14 a 19 anos.

O programa SPSS (for Windows ${ }^{\circledR}$ versão 22.0) foi utilizado para análise estatística. A distribuição dos dados foi realizada por meio do teste Kolmogorov-Smirnov. Já para a comparação das médias, utilizou-se do teste t de Student ou Mann-Whitney de acordo com a distribuição das variáveis.

A associação entre as variáveis dependentes e as explanatórias foi verificada por meio do cálculo do Odds Ratio (OR), utilizando a regressão logística binária. As variáveis que apresentaram valor de $\mathrm{p}<0,20$ na análise bivariada foram inseridas no modelo, sendo ajustadas para sexo, idade, renda familiar e tipo de escola. O nível de significância adotado para os testes foram de $\mathrm{p}<0,05$.

\section{RESULTADOS}

Dos 348 escolares selecionados, 21 foram excluídos - 11 por recusas e 10 por hemólise do sangue coletado -, totalizando uma amostra final de 327 adolescentes. As características deles estão apresentadas na Tabela 1, em que é possível verificar que $59,9 \%$ desses escolares eram do sexo feminino, com renda familiar menor que dois salários mínimos e estudantes da rede pública de ensino (65,7\%). Além disso, a prevalência da SM foi observada em $7 \%$ dos adolescentes.

As características antropométricas, bioquímicas e pressóricas da população do estudo são apresentadas na Tabela 2, na qual se pode notar que as variáveis como peso, CC, glicose, PAS e PAD foram significativamente maiores para o sexo masculino.

$\mathrm{Na}$ Tabela 3, são apresentadas as análises brutas e ajustadas. Pode-se observar que apenas a variável obesidade foi mantida para análise multivariada $(\mathrm{p}<0,20)$ e que houve uma associação significativa ( $<<0,03$; OR: 2,09) entre obesidade e prevalência de SM, apontando uma maior chance de um adolescente obeso apresentar SM.

Quanto à distribuição dos adolescentes de acordo com o número de componentes da SM, 51,5\% apresentaram um componente característico desse transtorno metabólico (Figura 1).

Tabela 1. Distribuição quantitativa das variáveis dos componentes da síndrome metabólica entre estudantes do ensino médio, na faixa etária de 14 a 19 anos, de escolas públicas e particulares do município de Teresina-PI, 2016

\begin{tabular}{lcc}
\multicolumn{1}{c}{ Variáveis } & N & $\%$ \\
Sexo & & \\
Masculino & 131 & $40,1 \%$ \\
Feminino & 196 & $59,9 \%$ \\
Tipo de escola & & \\
Particular & 112 & $34,3 \%$ \\
Pública & 215 & $65,7 \%$ \\
Renda familiar & & \\
<2 SAM & \\
>2 SAM & & $70,9 \%$ \\
Síndrome metabólica & 95 & 29,1 \\
Presença & & \\
Ausência & 24 & $7,0 \%$ \\
\hline
\end{tabular}

*SAM: Salário Mínimo Mensal 
Tabela 2. Valores médios e desvios-padrão das características antropométricas e bioquímicas dos adolescentes provenientes de escolas públicas e particulares do município de Teresina-PI, segundo sexo e população total, 2016

\begin{tabular}{|c|c|c|c|}
\hline \multirow{2}{*}{ Sexo } & Masculino (n) & Feminino (n) & Total $(n=328)$ \\
\hline & Média \pm DP & Média \pm DP & Média \pm DP \\
\hline Idade (anos) & $16,6 \pm 1,3$ & $16,4 \pm 1,2$ & $16,5 \pm 1,2$ \\
\hline Peso $(\mathrm{kg})^{*}$ & $64,6 \pm 12,6^{\mathrm{b}}$ & $54,8 \pm 10,2^{\mathrm{a}}$ & $58,8 \pm 12,2$ \\
\hline Altura $(\mathrm{cm})$ & $180,8 \pm 2,1$ & $181,1 \pm 2,1$ & $180,9 \pm 1,3$ \\
\hline $\operatorname{IMC}\left(\mathrm{kg} / \mathrm{m}^{2}\right)$ & $21,7 \pm 3,5$ & $21,3 \pm 3,6$ & $21,5 \pm 3,5$ \\
\hline $\mathrm{CC}(\mathrm{cm})^{*}$ & $76,1 \pm 10,7^{b}$ & $71,2 \pm 8,6^{\mathrm{a}}$ & $73,2 \pm 9,7$ \\
\hline HDL-c (mg/dL) & $14,9 \pm 19,5$ & $16,5 \pm 21,1$ & $15,9 \pm 20,4$ \\
\hline TG (mg/dL) & $73,5 \pm 33,2$ & $74,2 \pm 36,4$ & $73,9 \pm 35,1$ \\
\hline $\mathrm{CT}(\mathrm{mg} / \mathrm{dL})$ & $161,5 \pm 41,9$ & $165,0 \pm 34,2$ & $163,7 \pm 37,4$ \\
\hline Glicose $(\mathrm{mg} / \mathrm{dL})^{\star}$ & $94,9 \pm 22,7^{b}$ & $85,9 \pm 16,7^{\mathrm{a}}$ & $89,5 \pm 19,8$ \\
\hline PAS $(\mathrm{mmHg})^{*}$ & $116,7 \pm 1^{b}$ & $108,5 \pm 8,4^{\mathrm{a}}$ & $111,9 \pm 10,3$ \\
\hline $\operatorname{PAD}(\mathrm{mmHg})^{*}$ & $75,5 \pm 9,0^{\mathrm{b}}$ & $70,4 \pm 7,4^{\mathrm{a}}$ & $72,4 \pm 8,5$ \\
\hline
\end{tabular}

IMC: índice de massa corporal; CC: circunferência da cintura; TG: triglicerídeos; CT: colesterol total; PAD: pressão arterial diastólica; PAS: pressão arterial sistólica; DP: desvio-padrão. Letras diferentes na mesma linha significam diferença estatística; Teste t de Student. ${ }^{*}$ Teste Mann-Whitney, p $<0,05$

Tabela 3. Análise bruta e ajustada entre o estado nutricional e a síndrome metabólica em adolescentes provenientes de escolas públicas e particulares do município de Teresina-PI, 2016

\begin{tabular}{ccc} 
IMC $\left(\mathbf{k g} / \mathbf{m}^{2}\right)$ & Análise bruta & \multicolumn{1}{c}{ Análise ajustada $^{*}$} \\
\cline { 2 - 3 } Eutrofia/magreza & OR $(\mathbf{I C 9 5 \% )} \boldsymbol{p}(\mathrm{IC95 \% )} \boldsymbol{p}$ & 1 \\
Sobrepeso & 1 & $1,70(0,53-5,43) 0,37$ \\
Obesidade & $1,76(0,56-5,57) 0,33$ & $2,09(1,04-4,19) 0,03$ \\
\hline
\end{tabular}

IMC: índice de massa corporal; OR: odds ratio; IC95\%: intervalo de confiança. ${ }^{*}$ Análise ajustada por sexo, idade, renda familiar e tipo de escola

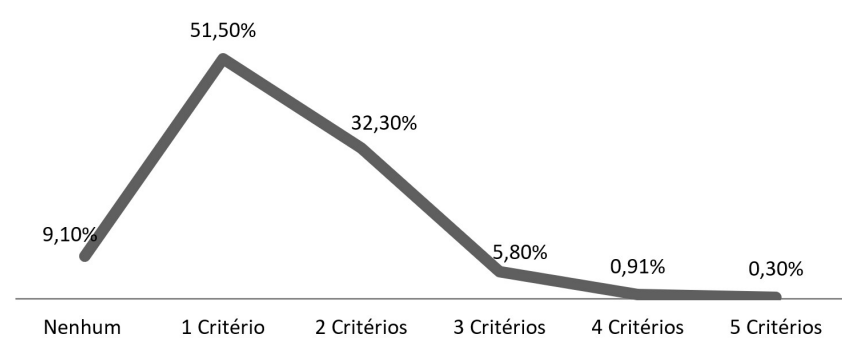

Figura 1. Distribuição do quantitativo dos critérios da síndrome metabólica entre estudantes do ensino médio, na faixa etária de 14 a 19 anos, de escolas públicas e particulares do município de Teresina-PI, 2016

\section{DISCUSSÃO}

Atualmente, há um aumento mundial de crianças e adolescentes que se encontram com excesso de peso, e, assim, paralelamente acontece uma maior prevalência dos casos de $\mathrm{SM}^{27}$. Neste estudo, a prevalência encontrada foi de $7 \%$, semelhante à prevalência mundial comparada com outras pesquisas, pois, de acordo com o critério da NCEP/ATP III ${ }^{9}$ com adaptações para adolescentes, observou-se na literatura uma prevalência global que varia de $2,5 \%$ a $22,2 \%$.

As definições da SM, propostas pela OMS, NCEP/ATP III e IDF, têm ocasionado divergências nas prevalências entre adolescentes. Os resultados apresentados por Neto et al. ${ }^{28}$ nas cidades do Paraná, com uma prevalência dessa síndrome de
6\% em São Mateus do Sul e 6,7\% na cidade de Jacarezinho, corroboram os dados encontrados na presente pesquisa. Entretanto, Kuschniri et al. ${ }^{12}$ realizaram recentemente um levantamento no Brasil, no qual reuniram cerca de $37 \mathrm{mil}$ adolescentes, e constataram uma prevalência de 2,6\% dessa síndrome. As divergências entre as prevalências observadas nas pesquisas podem ser explicadas em parte pelas diferenças nos pontos de corte e amostragens utilizados ${ }^{29}$, pois existem diferenças entre as regiões por causa de fatores genéticos, estilo de vida e hábitos alimentares.

As características da população foram semelhantes às relatadas por Kuschniri et al. ${ }^{12}$, em que a maioria dos adolescentes analisados era do sexo feminino e estudantes de escolas públicas. Além disso, peso, CC $(\mathrm{cm})$, PAS e PAD foram significativamente maiores $(\mathrm{p}<0,05)$ para o sexo masculino. Os dados pressóricos encontrados foram semelhantes aos do estudo de Song et al..$^{30}$ realizado com adolescentes, no qual os níveis de PAS e PAD estiveram maior no sexo masculino.

O sobrepeso e a obesidade estão diretamente relacionados ao desenvolvimento da SM e de seus marcadores isolados; portanto, o controle do peso corporal torna-se importante durante a adolescência ${ }^{31}$. No Brasil, segundo dados da Pesquisa Nacional de Saúde do Escolar, o excesso de peso esteve presente em $23,7 \%$ dos adolescentes, e a obesidade, em 7,8\% ${ }^{32}$. Esses resultados tornam-se pertinentes por mostrar que a obesidade 
está associada aos demais componentes da $\mathrm{SM}^{8}$. As análises realizadas neste estudo evidenciam essa associação entre a obesidade com a SM, demonstrando o dobro do risco para a SM nos adolescentes obesos, o que é preocupante pelo fato de se tratar de um público jovem e mostrar o reflexo dos atuais hábitos alimentares na saúde ${ }^{33}$.

Rossini et al. ${ }^{11}$, ao realizarem um estudo com escolares, também apresentaram resultados semelhantes ao desta pesquisa, ao perceberem um maior risco de SM em adolescentes obesos. A NCEP recomenda que a obesidade seja o alvo principal do tratamento da SM, pois a perda de peso melhora o perfil lipídico, reduz a pressão arterial, melhora a sensibilidade à insulina e reduz a glicose e o risco de doença aterosclerótica9 ${ }^{9}$.

Um maior número de componentes relacionados à SM mostra o risco aumentado de desenvolvimento de $\mathrm{DCV}^{8}$. No presente estudo, 51,5\% dos indivíduos apresentaram apenas um dos componentes, e $32,3 \%$, dois componentes. Considerando que a SM é um conjunto de alterações clínicas e bioquímicas, deve-se enfatizar que os indivíduos que já possuem um ou dois desses distúrbios provavelmente sofrerão dessa síndrome em um curto prazo, sendo necessárias intervenções não medicamentosas, tais como promoção de hábitos alimentares saudáveis e prática de atividade física, além de acompanhamento em serviços de saúde, a fim de contribuir para o controle de morbidades associadas à SM e para a manutenção da saúde global do indivíduo em longo prazo ${ }^{34}$.

Apesar dos progressos das pesquisas em relação à prevenção e ao tratamento da SM, ela continua sendo um importante problema de saúde pública. Por outro lado, estratégias de intervenções nutricionais têm demonstrado melhora nos parâmetros metabólicos e na qualidade de vida. Portanto, o presente estudo pode contribuir para futuras estratégias de intervenção efetiva para perda de peso entre adolescentes, reduzindo, assim, o risco de fatores que constituem a SM, tais como alterações no HDL-c, triglicerídeos e glicemia, que se fazem presente de forma crescente em adolescentes. No entanto, ainda há necessidade de estudos que mostrem se os resultados benéficos são mantidos após a intervenção.

Alguns pontos fortes importantes são considerados nesta pesquisa, como a amostra representativa dos adolescentes escolares, a utilização de critérios aceitos internacionalmente para diagnosticar a SM e a padronização das medidas antropométricas antes do início do estudo. Entretanto, o delineamento transversal, por causa da impossibilidade de determinar uma relação temporal de causalidade, e a definição de $\mathrm{SM}$, em razão da falta de uma referência padrão para os critérios de classificação dos adolescentes, são limitações deste estudo. Além disso, as medidas antropométricas também são outro critério limitante desta pesquisa, por causa dos vieses de medição e informação; portanto, com o objetivo de minimizar os erros, as medidas foram padronizadas e as equipes de avaliadores foram treinadas para melhorar a qualidade dos resultados do presente estudo.

A prevalência da SM apresentou-se elevada na população jovem do presente estudo, com importante associação entre a obesidade e a presença de SM. Outro fato preocupante foi que a maioria dos adolescentes possuía pelo menos um componente da síndrome. Assim, devem ser feitas intervenções nutricionais direcionadas à população mais jovem com intuito de realizar medidas que visem à melhoria dos hábitos alimentares e do estilo de vida.

\section{REFERÊNCIAS}

1. Martin K, Mani M. New targets to treat obesity and the metabolic syndrome. Eur J Pharmacol. 2015; 763: 64-74. http://dx.doi.org/10.1016/j. ejphar.2015.03.093.

2. Costa RF, Santos NS, Goldraich NP, Barski TF, Andrade KS, Kruel LMF. Síndrome metabólica em adolescentes obesos: comparação entre três diferentes critérios diagnósticos. J Pediatr (Rio J). 2012;88(4):303-9. http:// dx.doi.org/10.2223/JPED.2200. PMid:22622762.

3. Gobato AO, Vasquez AC, Zambo MP, Barros Filho AABR, Hessel G. Metabolic syndrome and insulin resistance in obese adolescents. Rev Paul de Pediatr. 2014; 32(1): 55-62. http://dx.doi.org/10.1590/S0103-05822014000100010.

4. Al-Hamad D, Raman V. Metabolic syndrome in children and adolescents. Transl Pediatr. 2017;6(4):397-407. http://dx.doi.org/10.21037/tp.2017.10.02. PMid:29184820.

5. Lira Neto JCG, Xavier MA, Borges JWP, Araújo MFM, Damasceno MMC, Freitas RWJF. Prevalence of Metabolic Syndrome in individuals with Type 2 Diabetes Mellitus. Rev Bras Enferm. 2017;70(2):265-70. http://dx.doi. org/10.1590/0034-7167-2016-0145. PMid:28403288.

6. Mohammadi SG, Mirmiran P, Bahadoran Z, Mehrabi Y, Azizi F. The association of dairy intake with metabolic syndrome and its components in adolescents: Tehran Lipid and Glucose Study. Int J Endocrinol Metab. 2015;13(3):e25201. http://dx.doi.org/10.5812/ijem.25201v2. PMid:26425126.

7. Vergetaki A, Linardakis M, Papadaki A, Kafatos A. Presence of metabolic syndrome and cardiovascular risk factors in adolescents and University students in Crete (Greece), according to different levels of snack consumption. Appetite. 2011;57(1):278-85. http://dx.doi.org/10.1016/j.appet.2011.05.309. PMid:21640150.

8. Wang Q, Yin J, Xu L, Cheng H, Zhao X, Xiang H, et al. Prevalence of metabolic syndrome in a cohort of Chinese schoolchildren: comparison of two definitions and assessment of adipokines as components by factor analysis. BMC Public Health. 2013;13(1):249. http://dx.doi.org/10.1186/14712458-13-249. PMid:23514611.

9. Expert Panel on Detection, Evaluation, and Treatment of High Blood Cholesterol in Adults. Executive summary of the third report of the National Cholesterol Education Program (NCEP) Expert Panel on Detection, Evaluation, And Treatment of High Blood Cholesterol in Adults (Adult Treatment Panel III). JAMA. 2001;285(19):2486-97. http://dx.doi. org/10.1001/jama.285.19.2486. PMid:11368702.

10. Cantalice ASC, Santos NCCB, Oliveira RC, Collet N, Medeiros CCM. Persistência da síndrome metabólica em crianças e adolescentes com 
excesso de peso de acordo com dois critérios diagnósticos: um estudo longitudinal. Medicina. 2015; 48(4): 342-8. http://dx.doi.org/10.11606/ issn.2176-7262.v48i4p342-348.

11. Rosini N, Moura SAZO, Rosini RD, Machado MJ, Silva EL. Síndrome metabólica e importância das variáveis associadas em crianças e adolescentes de Guabiruba-SC, Brasil. Arq Bras Cardiol. 2015;105(1):37-44. http://dx.doi. org/10.5935/abc.20150040. PMid:25993484.

12. Kuschniri MCC, Bloch KV, Szklo M, Klein CH, Barufaldi LA, Abreu GA, et al. ERICA: prevalência de síndrome metabólica em adolescentes brasileiros. Rev Saude Publica. 2016;50(Supl. 1):11s. http://dx.doi.org/10.1590/S015188787.2016050006701.

13. Brasil. Ministério da Saúde. Resolução n466/12. Conselho Nacional de Pesquisa com Seres Humanos. Diário Oficial da União, Brasília, 12 de dezembro de 2012.

14. Luiz ES, Torres TG, Hagnaninih MMF. Planejamento amostral. In: Luiz RR, Costa AJL, Nadanovsky P. Epidemiologia e bioestatística na pesquisa odontológica. São Paulo: Atheneu; 2005.

15. Brasil. Instituto Nacional de Estudos e Pesquisas Educacionais Anísio Teixeira. Educação Básica. Censo Escolar [Internet]. 2014. [citado em 2015 Jun 14] Disponível em: http://www.dataescolabrasil.inep.gov.br/ dataEscolaBrasil/home.seam</eref> Acesso em 14 jun. 2015.

16. Bloch KV, Klein CH, Szklo IM, Kuschnir MCC, Abreu GA, Barufaldi LA. Erica: Prevalências de hipertensão arterial e obesidade em adolescentes brasileiros. Rev Saude Publica. 2016;50(Supl. 1):9s. http://dx.doi.org/10.1590/ s01518-8787.2016050006685. PMid:26910553.

17. Armitage P. Statistical method in medical research. New York: John Wiley and Sons; 1981.

18. World Health Organization. WHO child growth standards: length/heightforage, weight-for-age, weight-for-length, weight-for height and body mass index-for-age: methods and development. Geneva: World Health Organization; 2007.

19. Callaway CW. Circunferences. In: Lohman TG, Roche AF, Martorell R, editors. Anthropometric standardization reference manual. Champaign: Human Kinetics; 1988. p. 39-54.

20. Fredriks AM, Van Buuren S, Verloove-Vanhorick MFSP, Wit JM. Are age references for waist circumference, hip circumference and waist-hip ratio in Dutch children useful in clinical practice? Eur J Pediatr. 2005;164(4):21622. http://dx.doi.org/10.1007/s00431-004-1586-7. PMid:15662504.

21. Malachias MVB, Souza WKSB, Plavnik FL, Rodrigues CIS, Brandão AA, Neves MFT, et al. 7a Diretriz Brasileira de Hipertensão Arterial. Arq Bras Cardiol. 2016;107(3 Supl):1-83. PMid:27819380.

22. Molina MCB, Faria CP, Montero MP, Cade NV, Mill JG. Fatores de risco cardiovascular em crianças de 7 a 10 anos de área urbana, Vitória, Espírito Santo, Brasil. Cad Saude Publica. 2010;26(5):909-17. http://dx.doi.org/10.1590/ S0102-311X2010000500013. PMid:20563391.
23. Christofaro DGD, Ritti-Dias RM, Fernades RA, Polito MD, Andrade SM, Cardoso JR, et al. Detecção de hipertensão arterial em adolescentes através de marcadores gerais e adiposidade abdominal. Arq Bras Cardiol. 2011;96(6):465-70. http://dx.doi.org/10.1590/S0066-782X2011005000050. PMid:21537530.

24. Friedewald WT, Levy RI, Fredrickson DS. Estimation of the concentration of low-density lipoprotein cholesterol in plasma, without use of the preparative ultracentrifuge. Clin Chem. 1972;18(6):499-502. PMid:4337382.

25. Faludi AA, Izar MCO, Saraiva JFK, Chacra APM, Bianco HT, Afiune Neto A, et al. Atualização da Diretriz Brasileira de Dislipidemias e Prevenção da Aterosclerose - 2017. Arq Bras Cardiol. 2017;109(1 Supl. 1):1-76. http:// dx.doi.org/10.5935/abc.20170121.

26. American Diabetes Association. Diagnosis and classification of diabetes mellitus. Diabetes Care. 2010;33(Suppl. 1):S62-9. http://dx.doi.org/10.2337/ dc10-S062.

27. Enes RP, Gutiérrez BC, Gil NA, Martín-Frías M, Blanco MA, Castellanos RB. Influencia étnica em la prevalência de síndrome metabólico em la poblacion pediátrica obesa. Pediatr (Barc). 2012;1(6):75-80. http://dx.doi. org/10.1016/j.anpedi.2012.04.010.

28. Neto CGL, Bozza R, Ulbrich A, Mascarenhas LPG, Boguszewski MCS, Campos W. Síndrome metabólica em adolescentes de diferentes estados nutricionais. Arq Bras Endocrinol Metabol. 2012;56(2). http://dx.doi. org/10.1590/S0004-27302012000200003.

29. Pontes LM, Amorim RJM, Lira PIC. Componentes da síndrome metabólica e fatores associados em adolescentes: estudo caso-controle. Revista da AMRIGS. 2016;60(2):121-8.

30. Song S, Paik HY, Song WO, Song Y. Metabolic syndrome risk factors are associated with white rice intake in Korean adolescent girls and boys. $\mathrm{Br} \mathrm{J}$ Nutr. 2015; 113 (3): 479-87. https://doi.org/10.1017/S0007114514003845.

31. Pegolo GE. Síndrome Metabólica: aspectos clínicos e fatores modificáveis associados ao seu desenvolvimento em adolescentes - revisão. Segur Aliment Nutr. 2012;19(2):63-72. http://dx.doi.org/10.20396/san.v19i2.8634611.

32. Brasil. Ministério da Saúde. Pesquisa Nacional de Saúde do Escolar (PENSE) [Internet]. Rio de Janeiro: IBGE; 2015 [citado em 2015 Jun 10]. Disponível em: https://biblioteca.ibge.gov.br/visualizacao/livros/liv97870.pdf

33. Barroso TA, Marins LB, Alves R, Gonçalvez ACS, Barroso SG, Rocha GS. Associação entre a obesidade central e a incidência de doenças e fatores de risco cardiovascular. Int J Cardiovasc Sci. 2017;30(5):416-24. http:// dx.doi.org/10.5935/2359-4802.20170073.

34. Simão AF, Precoma DB, Andrade JP, Correa Filho H, Saraiva JFK, Oliveira GMM, et al. I Diretriz Brasileira de Prevenção Cardiovascular. Arq Bras Cardiol. 2013;101(6 Supl. 2):1-63. http://dx.doi.org/10.5935/abc.2013S012. PMid:24554026.
Recebido em: Maio 23, 2018 Aprovado em: Abr. 05, 2019 